\title{
Kostentreiberanalyse am Beispiel der Deutschen Lufthansa AG
}

\section{Teil 1: Aufgabenstellung}

\author{
Debbie Claassen, Christian Hofmann und Nina Kühne
}

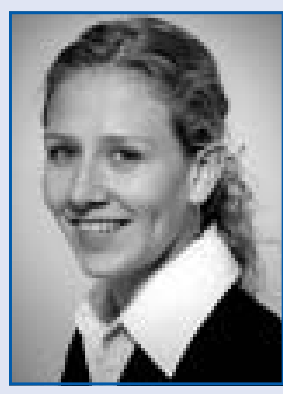

Debbie Claassen, M.Sc., ist wissenschaftliche Mitarbeiterin und Doktorandin am Institut für Unternehmensrechnung und Controlling der Ludwig-MaximiliansUniversität München.

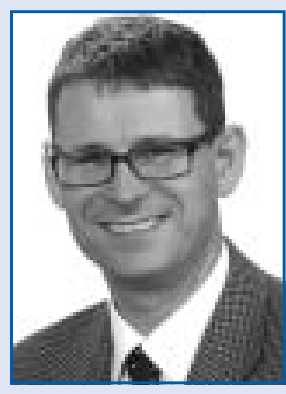

Prof. Dr. Christian Hofmann ist Professor am Institut für Unternehmensrechnung und Controlling der Ludwig-MaximiliansUniversität München.

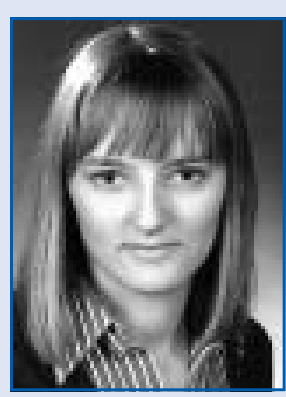

Nina Kühne, B.Sc., ist wissenschaftliche Mitarbeiterin und Doktorandin am Institut für Unternehmensrechnung und Controlling der Ludwig-MaximiliansUniversität München.

Treibstoffkosten stellen einen wesentlichen Kostenfaktor für Unternehmen der Luftfahrtbranche dar. Aus diesem Grund ist es gerade für diese Unternehmen besonders wichtig, eine präzise Kostentreiberplanung zu erstellen. In dieser Fallstudie werden verschiedene Verfahren angewandt, um am Beispiel der Deutschen Lufthansa $A G$ eine Kostentreiberplanung durchzuführen.

Stichwörter: Treibstoffkosten, Kostentreiber, lineare Kostenfunktionen, Multikollinearität, Data Envelopment Analysis

\section{Problemstellung und Fallbeschreibung}

„Gewicht sparen - Airline stellt nur Frauen ein“ (vgl. Die Welt, Juli 2013).

Diese Schlagzeile stammt aus einem kürzlich erschienen Artikel der Zeitung Die Welt. In diesem wird über eine indische Fluggesellschaft berichtet, die eine 100-prozentige Frauenquote anstrebt. Grund dafür ist das im Durchschnitt 15 bis 20 Kilogramm geringere Gewicht des weiblichen Geschlechts und damit auch der daraus folgende geringere Treibstoffverbrauch. Doch dies ist nicht die einzige Maßnahme zur Treibstoffreduzierung durch Gewichtsreduzierung, die die indische Fluggesellschaft aufgesetzt hat. Auch die Größe der Bordmagazine und die Fülle der Bordwassertanks wurden reduziert zur Senkung des Treibstoffverbrauchs.

Dieser Artikel zeigt die anscheinend große Bedeutung des Gewichts für den Treibstoffverbrauch. Doch wie kann das Gewicht eines Flugzeuges geschätzt werden und welche weiteren Größen haben einen Einfluss auf die Höhe der Treibstoffkosten? Diese und noch weitere Fragen sollen in den folgenden Aufgaben beantwortet werden. Dafür werden Verkehrsdaten der Deutschen Lufthansa AG zur Analyse herangezogen. Im Jahr 2012 stellte der Materialaufwand der Deutschen Lufthansa AG bereits etwa $55 \%$ des Gesamtaufwandes dar, wobei Treibstoffkosten alleine schon $40 \%$ des Materialaufwandes ausmachten. Diese Zahlen zeigen deutlich, dass Treibstoffkosten ein großer Kostenfaktor für das Flugunternehmen sind. Aus diesem Grund ist es wichtig, dass das Unternehmen eine präzise Kostenplanung durchführt, in der die relevanten Kostentreiber identifiziert werden und ihr Einfluss auf die Gesamtkosten herausgearbeitet wird (vgl. Friedl et al., 2013, S. 235-237).

\section{Aufgaben}

Versetzen Sie sich in die Rolle des Kostenplaners und versuchen Sie anhand der folgenden Aufgaben eine präzise Kostentreiberplanung für die Aufwandsposition Treibstoffkosten darzustellen.

Zum Lösen der Aufgaben b, c, d, f, g und h steht eine Excel-Tabelle auf der WiSt-Homepage unter www.beckshop.de/cbb zum Download bereit. 


\section{Aufgabe a)}

Warum können Treibstoffkosten für den Passagierverkehr durch folgende Einflussfaktoren approximiert werden: Anzahl Flüge, angebotene Sitzkilometer, Anzahl Fluggäste (vgl. Banker/Johnston, 1993, S. 576-601; Dikolli/Sedatole, 2004, S. 345-359; Friedl et al., 2013, S. 235-237)?

\section{Aufgabe b)}

Im Folgenden wird die Kostensituation der Deutschen Lufthansa AG im Bereich Passagierverkehr näher analysiert. Verwenden Sie dafür die Daten für den Passagierverkehr aus der Excel-Tabelle (Treibstoffkosten Passagierverkehr, Anzahl Fluggäste, Anzahl Flüge Passagierverkehr und Angebotene Sitzkilometer). Untersuchen Sie die Entwicklung der Treibstoffkosten über die Zeit und den $\mathrm{Zu}-$ sammenhang der jeweiligen unabhängigen Variablen und der abhängigen Variablen Treibstoffkosten im Passagierverkehr, indem Sie die nötigen Plots erstellen. Interpretieren Sie Ihre Ergebnisse.

Hinweis:

In dieser Aufgabe sind vier Plots in Excel zu erstellen (Entwicklung der Treibstoffkosten im Passagierverkehr über die Zeit, der Einfluss der Variable „Anzahl Fluggäste“ auf „Treibstoffkosten Passagierverkehr“, der Einfluss der Variable „Anzahl Flüge Passagierverkehr“ auf „Treibstoffkosten Passagierverkehr" und der Einfluss der Variable „Angebotene Sitzkilometer“ auf „Treibstoffkosten Passagierverkehr").

\section{Aufgabe c)}

Lineare Kostenfunktionen zur Ermittlung des Einflusses einer unabhängigen Variablen auf eine abhängige Variable können mit Hilfe der Hoch-Tief-Methode oder der linearen Regression spezifiziert werden. Zur Bestimmung einer linearen Kostenfunktion benötigt man jeweils Achsenabschnitt und Steigung.

Im Rahmen der Hoch-Tief-Methode berechnet man Steigung und Achsenabschnitt der linearen Kostenfunktion über folgende Formeln, wobei $x$ und $y$ die unabhängige bzw. abhängige Variable darstellen (vgl. Friedl et al., 2013, S. 217-219):

$$
\begin{aligned}
& \text { Kostendifferenz bei maximaler und minimaler } \\
& \text { Ausprägung der unabhängigen Variable }
\end{aligned}
$$

Die Koeffizienten der linearen Regression (Achsenabschnitt und Steigung der linearen Kostenfunktion) können sowohl über entsprechende Formeln (z. B. in Excel) als auch über eine Analysefunktion in Excel berechnet werden. Steigung $b$ und Achsenabschnitt $a$ der Regressionsgleichung können über folgende Formeln berechnet werden (vgl. Friedl et al., 2013, S. 220-221, 233-235): $b=\frac{\sum_{i=1}^{n}\left(x_{i}-\bar{x}\right)\left(y_{i}-\bar{y}\right)}{\sum_{i=1}^{n}\left(x_{i}-x\right)^{2}}$

$a=\bar{y}-b \bar{x}$

$\bar{x}$ : Mittelwert der unabhängigen Variable $x_{i}$

$\bar{y}$ : Mittelwert der abhängigen Variable $y_{i}$

Schätzen Sie den Einfluss der in Aufgabenteil a) genannten Faktoren auf die Treibstoffkosten im Passagierverkehr mit Hilfe der Hoch-Tief-Methode. Berechnen Sie anschließend beispielhaft den Einfluss der Variable „Anzahl Fluggäste" auf die Treibstoffkosten im Passagierverkehr mit der oben genannten Formel in Excel. Führen Sie anschließend die entsprechenden einfachen linearen Regressionen in Excel durch zur Schätzung des Einflusses jeder unabhängigen Variablen auf die abhängige Variable „Treibstoffkosten Passagierverkehr" und vergleichen Sie Ihre Ergebnisse.

Interpretieren Sie anschließend Ihre Ergebnisse und erläutern Sie Vor- und Nachteile der beiden Methoden.

Hinweis:

Zur Erstellung einer linearen Regression in Excel muss man zunächst das Add-In „Analyse-Funktionen“ in Excel aktivieren.

Aufgabe d)

Kann anhand dieser Daten eine präzise multiple lineare Regression durchgeführt werden?

Hinweis: Diese Frage zielt auf das Problem der Multikollinearität ab.

Erläutern Sie Ihre Antwort anhand folgender Überprüfungen (vgl. Auer, 2011; Eckey et al., 2011; Greene, 2011; Gujarati, 2007; Hackl, 2012; Kleiber/Zeileis, 2008; Maddala/Lahiri, 2009; Studenmund, 2001; Wooldridge, 2006):

\section{Einfache Korrelationsanalyse}

Im Rahmen der einfachen Korrelationsanalyse können hier die Bravais-Pearson-Korrelationskoeffizienten zwischen den drei unabhängigen Variablen (Anzahl Fluggäste, Anzahl Flüge Passagierverkehr, Angebotene Sitzkilometer) berechnet werden. Die entsprechenden Korrelationsmatrizen lassen sich sehr einfach in der Datenanalyse in Excel berechnen. (Multikollinearität falls $r_{j k}>0,8$;

$r_{j k}$ : Korrelationskoeffizient der Variable j und k)

\section{Erweiterte Korrelationsanalyse}

Der multiple Korrelationskoeffizient für die erweiterte Korrelationsanalyse wird im Rahmen der multiplen linearen Regression ermittelt. (Multikollinearität falls $r_{j k}>R_{j k l}$; $R_{j k l}:$ multipler Korrelationskoeffizient der Variablen j, k und 1) 


\section{Determinante}

Bei dieser Methode benötigt man zunächst die Korrelationsmatrix R der unabhängigen Variablen. Anschließend kann in Excel die Determinante dieser Matrix berechnet werden.

(Multikollinearität falls $|R| \approx 0 ;|R|$ : Determinante der Korrelationsmatrix R)

\section{Verfahren der Hilfsregressionen}

Bei diesem Verfahren werden Regressionen auf Basis der Daten der unabhängigen Variablen durchgeführt, indem jeweils eine der unabhängigen Variablen als abhängige Variable eingesetzt wird und die übrigen unabhängigen Variablen weiterhin die unabhängigen Variablen darstellen. Dadurch wird getestet, ob bereits zwischen den unabhängigen Variablen ein signifikant linearer Zusammenhang besteht. Zur Ermittlung der Koeffizienten kann das Regressionsanalysetool in Excel verwendet werden. Die entscheidenden Kennzahlen hier sind die F-Statistiken, die Toleranzkoeffizienten und die Varianzinflationsfaktoren:

tol $_{j}=1-R_{j, \text { adjusted }}^{2}$

$V I F_{j}=\frac{1}{t_{0 l}}$

(Multikollinearität falls $F>F_{K r i t}$; tol $_{j}<0,10 ; V I F_{j}>10$ )

\section{Aufgabe e)}

Wie könnte das Problem der Multikollinearität gelöst werden? Geben Sie Beispiele.

\section{Aufgabe f)}

Zum Vergleich der Effizienz der beiden Bereiche Cargound Passagierverkehr der Deutschen Lufthansa AG soll nun eine Effizienzanalyse durchgeführt werden. Als Basis für den Vergleich werden die Treibstoffkosten und die Anzahl der Flüge in den beiden Bereichen als Daten herangezogen.

Eine Effizienzanalyse kann mit Hilfe der Data Envelope Analysis durchgeführt werden. Darunter versteht man eine Effizienzanalyse von vergleichbaren Entscheidungseinheiten. Allgemein sind Entscheidungseinheiten „Objekte“, die aus „Inputs“ „Outputs“ erzeugen. Damit verschiedene Entscheidungseinheiten bezüglich ihrer Effizienz beurteilt werden können, müssen Entscheidungseinheiten verwendet werden, die sich durch dieselben Inputs und Outputs auszeichnen. Falls mehrere Inputs und Outputs in die Analyse einbezogen werden sollen, werden diese mit entsprechenden „Bedeutungsgewichten“ versehen. Entscheidungskriterium ist die relative Effizienz der Entscheidungseinheiten, die sich bemisst aus dem Quotienten der tatsächlichen Effizienz und dem effizienten Rand (Data Envelope).

Zur Berechnung der relativen Effizienz müssen zunächst für jedes Quartal die tatsächlichen Effizienzkoeffizienten für beide Bereiche berechnet werden, indem die Treibstoffkosten jedes Quartals (abhängige Variable y) durch die zugehörige Anzahl an Flügen (unabhängige Variable $\mathrm{x}$ ) dividiert werden. Aus den gewonnenen Effizienzkoeffizienten beider Bereiche wird im nächsten Schritt das Minimum (der effiziente Rand) ausgewählt. Die relative Effizienz berechnet sich anschließend mit folgender Formel (vgl. Barr et al., 2004, S. 539-566; Charnes et al., 1978, S. 429-444; Charnes et al., 1985, S. 91-107; Coelli/Rao, 1989; Cooper et al., 2007).

Relative Effizienz $=\frac{\frac{y}{x}}{\min \left(\frac{y}{x}\right)}$

Analysieren Sie mit Hilfe der Data Envelope Analysis, ob der Cargo-Bereich oder der Passagierverkehr der Deutschen Lufthansa AG in Bezug auf die Anzahl an Flügen den Treibstoff effizienter einsetzt und interpretieren Sie Ihre Ergebnisse.

Hinweis:

Zur Lösung dieser Aufgabe ist es sinnvoll, die relative Effizienz der beiden Bereiche über die Quartale in einem Diagramm zu plotten.

\section{Aufgabe g)}

Abb. 1 zeigt die Entwicklung der Güterpreise von 2005 bis 2013.

Wie man deutlich erkennen kann, gab es, abgesehen von einem Quartal, in allen Quartalen eine Inflation in Deutschland. Natürlich wirkt sich eine Erhöhung der Güterpreise auch auf die Höhe der Treibstoffkosten aus (vgl. Friedl et al., 2013, S. 236). Hier ist nun die Frage, wie sich die Regressionskoeffizienten aus den einfachen linearen Regressionen ändern, wenn inflationsbereinigte Treibstoffkosten im Passagierverkehr verwendet werden. Verwenden Sie für die Berechnung der inflationsbereinigten Treibstoffkosten im Passagierverkehr die angegebenen Inflationsraten der Quartale von 2005 bis 2013 und die ursprünglichen Treibstoffkosten im Passagierverkehr. Führen Sie anschließend zwei Regressionen durch, die den Einfluss der Variable „Anzahl Fluggäste“ bzw. „Anzahl Flüge Passagierverkehr“ auf die abhängige Variable „Inflationsbereinigte Treibstoffkosten Passagierverkehr" zeigen. Interpretieren Sie Ihre Ergebnisse. Warum ist der Einfluss der Variable „Angebotene Sitzkilometer“ auf die Variable „Inflationsbereinigte Treibstoffkosten Passagierverkehr" nicht interessant?

\section{Aufgabe h)}

Intuitiv müssten auch die Kerosinpreise entscheidend für die Höhe der Treibstoffkosten sein (vgl. Friedl et al., 2013, S. 236). Führen Sie unter Zuhilfenahme der Kerosinpreise der letzten Jahre eine geeignete Regression zur Überprüfung dieser Hypothese durch. Untersuchen Sie außerdem auf Basis der Daten aus dem Passagierverkehr, welchen 


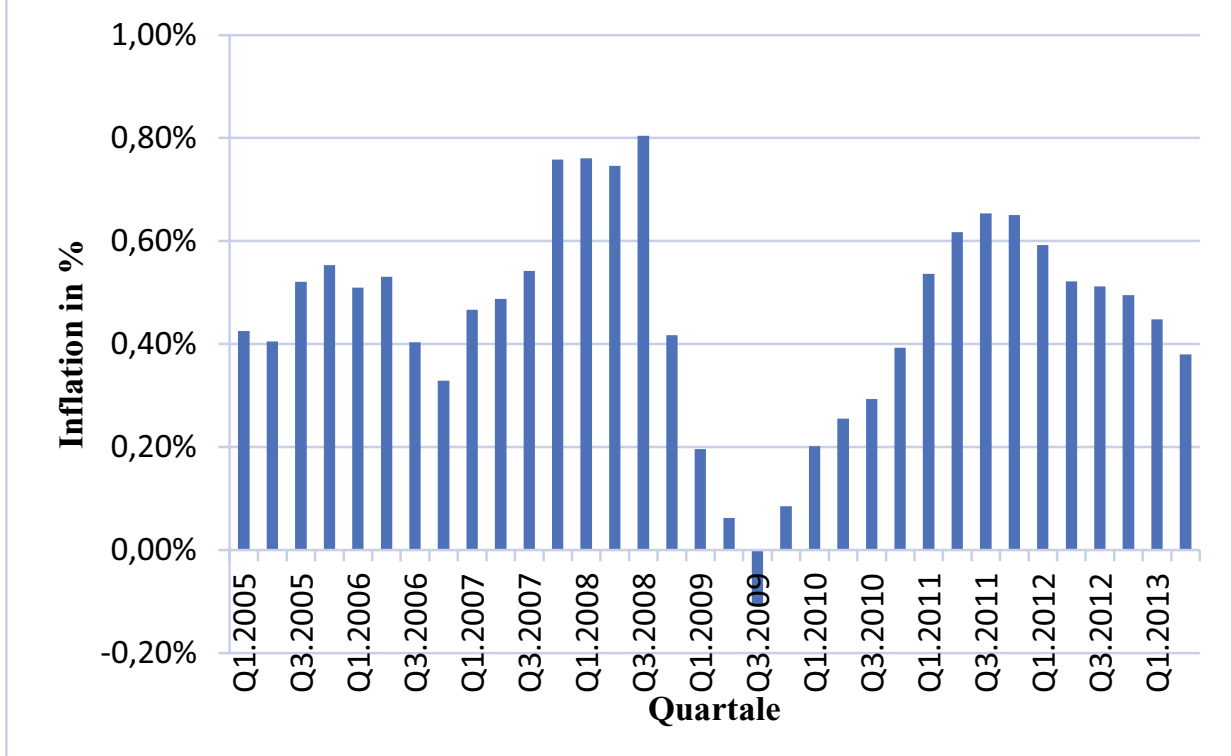

Abb. 1: Inflation pro Quartal

Einfluss die jeweiligen unabhängigen Variablen auf die abhängige Variable haben, wenn der Kerosinpreis als zweite unabhängige Variable in die Regressionsgleichung aufgenommen wird. Interpretieren Sie Ihre Ergebnisse.

Die Lösungen zu den Aufgaben finden sich im WiSt-Heft, Nr. 2 (2015), S. 112-117.

\section{Literatur}

Auer, L. von, Ökonometrie, 5. Aufl., Berlin 2011.

Barr, R., W. Cooper, L. Seiford, J. Zhu, Dea Software Tools and Technology, Handbook on Data Envelopment Analysis, in: International Series in Operations Research \& Management Science, Vol. 71 (2004), S. 539-566.

Banker, R.-D., H.-H. Johnston, An Empirical Study of Cost Drivers in the U.S. Airline Industry, in: The Accounting Review, Vol. 68, No. 3 (1993), S. 576-601.

Charnes, A., W.-W. Cooper, B. Golany, L. Seiford, J. Stutz, Foundations of Data Envelopment Analysis for Pareto-Koopmans efficient empirical production functions, in: Journal of Econometrics, Vol. 30 (1985), S. 91-107.

Charnes, A., W.-W. Cooper, E. Rhodes, Measuring the efficiency of decision making units, in: European Journal of Operational Research, Vol. 2 (1978), S. 429-444.
Coelli, T., P. Rao, Introduction to efficiency and productivity analysis, Boston 1989.

Cooper W.-W., L.-M. Seiford, K. Tone, Data Envelopment Analysis - A Comprehensive Text with Models, Applications, References and DEA-Solver Software, Boston/Dordrecht/London 2007.

Dikolli, S.-S., K.- L. Sedatole, Delta's New Song: A Case on Cost Estimation in the Airline Industry, in: Issues in Accounting Education, Vol. 19, No. 3 (2004), S. 345-358.

Eckey, H.-F., R. Kosfeld, C. Dreger, Ökonometrie, 4. Aufl., Wiesbaden 2011.

Friedl, G., C. Hofmann, B. Pedell, Kostenrechnung, 2. Aufl., München 2013.

Greene, W.-H., Econometric Analysis, $7^{\text {th }}$ ed., Upper Saddle River 2011.

Gujarati, D.-N., Basic Econometrics, $4^{\text {th }}$ ed., New York 2007.

Hackl, P., Einführung in die Ökonometrie, 2. Aufl., München 2012.

Kleiber, C., A. Zeileis, Applied Econometrics with R, New York 2008.

Maddala, G.-S., K. Lahiri, Introduction to Econometrics, $4^{\text {th }}$ ed., Chichester 2009.

Studenmund, A.-H., Using Econometrics: A Practical Guide, 4th ed., Boston 2001.

Wooldridge, J.-M., Introductory Econometrics: A Modern Approach, $3^{\text {rd }}$ ed., Toronto 2006. 


\section{Aktuell - gebunden - zweisprachig.}

\section{IFRS komplett}

Diese gebundene IFRS-Textausgabe in Deutsch und Englisch ist das praktische und handliche Arbeitsmittel für alle, die sich mit internationaler Rechnungslegung befassen.

\section{Zur Neuauflage}

Die "Aktuellen IFRS-Texte 2014" enthalten alle von der Europäischen Union bis zum 1. Oktober 2014 offiziell übernommenen und im EU-Amtsblatt veröffentlichten internationalen Rechnungslegungsstandards (IFRS und IAS) einschließlich ihrer Interpretationen (IFRIC und SIC) und zugehörigen Anhänge sowie das Rahmenkonzept (Framework) jeweils synoptisch gegenüber gestellt in deutscher und englischer Sprache. Damit wird ein Vergleich mit dem englischen Ursprungstext ermöglicht, der in Auslegungsfragen herangezogen werden muss.

Basis sind die mit VO (EG) 1126/2008 in neuer Übersetzung bekannt gemachten internationalen Rechnungslegungsstandards.

Neu eingearbeitet wurden alle daran anschließenden Änderungen.

\section{Unentbehrlich für}

- Abschlussersteller und Abschlussprüfer

- Rechnungslegungsleiter und Internationale Bilanzbuchhalter

- Analysten und Controller

- Wissenschaft und Ausbildung

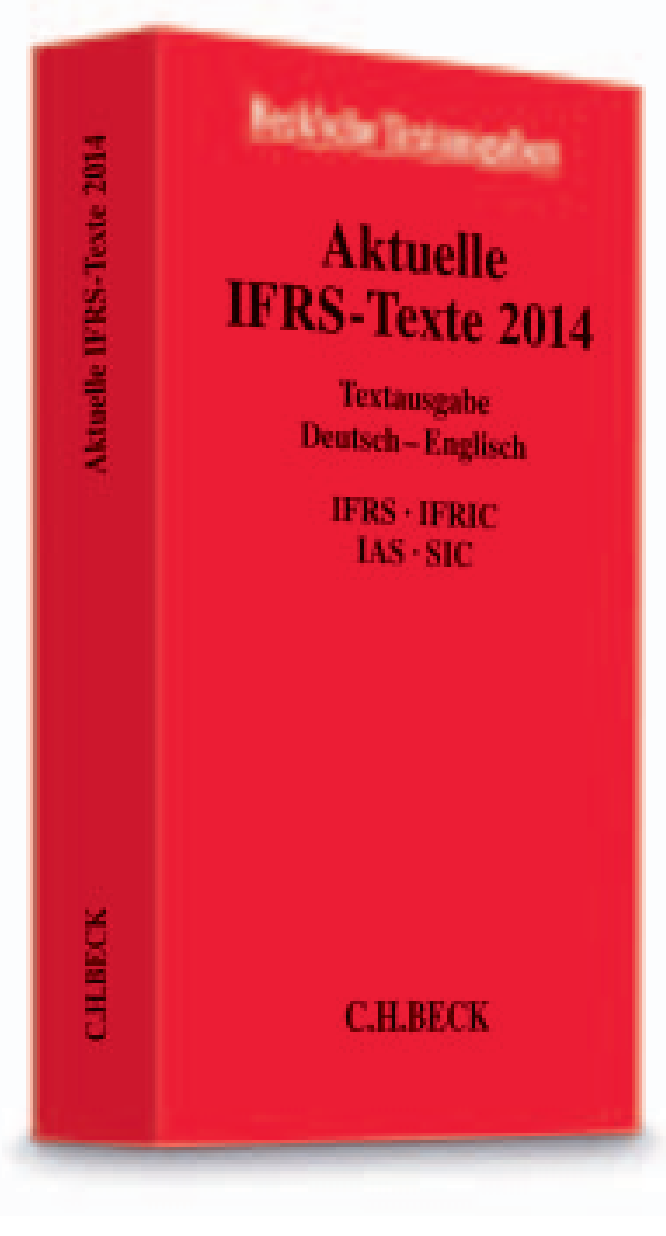

Aktuelle IFRS-Texte 2014

2014. XXVII, 2417 Seiten.

Kartoniert $€ 29,80$

ISBN 978-3-406-66939-2

Neu im Dezember 2014

Mehr Informationen:

www.beck-shop.de/bdztyq

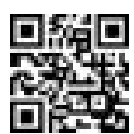




\section{So motivieren Sie Ihre Mitarbeiter.}

\section{Potenziale aktivieren}

Dieses Buch macht in leicht verständlicher Sprache mit den psychologischen Aspekten der Mitarbeitermotivation vertraut. Es zeigt auf,

- wie Sie die Motivation von Mitarbeitern beeinflussen können,

- wie Sie Tätigkeiten und Aufgaben gestalten können, sodass sie motivieren,

- welche Motivationshindernisse Sie beseitigen müssen,

- wie Sie durch Art der Führung und konstruktive Teamarbeit Leistungspotenziale erschließen,

- wie Sie Organisationen strukturieren sollten, damit sich die Leistungsmotivation entfaltet, und

- wie Sie sich selbst motivieren.

Eine Vielzahl anschaulicher Beispiele erleichtert die unmittelbare Übertragung der Motivationsfaktoren auf die Praxis der Mitarbeiterführung.

\section{Neu in der 5. Auflage}

- Selbstmanagement-Fähigkeiten trainieren

- Feindseliges Führungsverhalten die »dunkle Seite« der Führung

- Mit "Diversity“ und Altersheterogenität umgehen

- Die größten Fehler bei Fusionen und Übernahmen

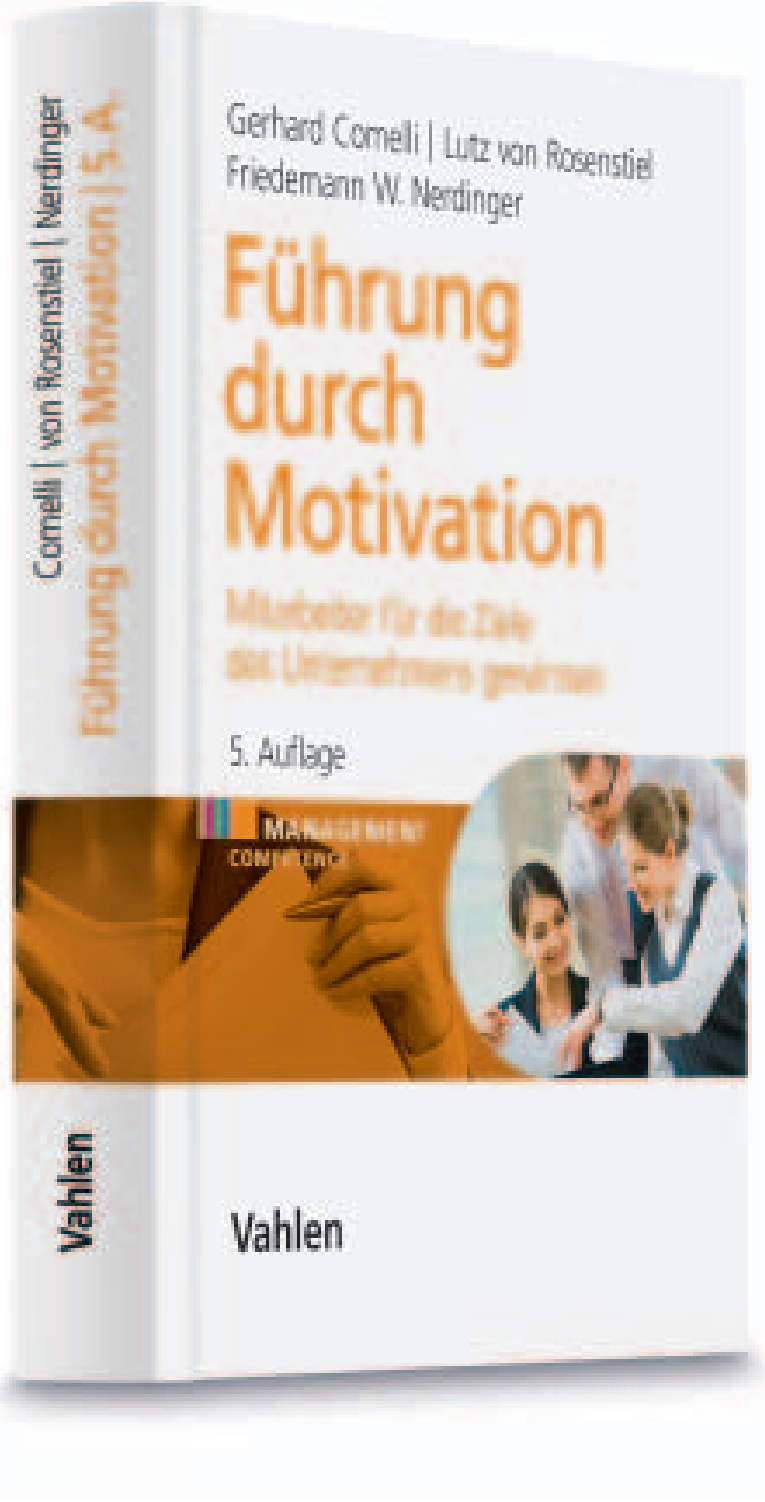

Comelli/von Rosenstiel/Nerdinger Führung durch Motivation

5. Auflage 2014. XII, 323 Seiten. Gebunden $€ 36,90$

ISBN 978-3-8006-4839-9

Neu im November 2014

Portofrei geliefert: vahlen.de/13656985 